University of Wollongong

Research Online

Faculty of Engineering and Information

Faculty of Engineering and Information

Sciences - Papers: Part A

Sciences

2015

Reconstructing highly accurate relative stopping powers in proton computed tomography

Blake Schultze

Baylor University

Paniz Karbasi

Baylor University

Valentina Giacometti

University of Wollongong,vg406@uowmail.edu.au

Tia E. Plautz

University of California, tiaplautz@gmail.com

Keith Schubert

Baylor University

See next page for additional authors

Follow this and additional works at: https://ro.uow.edu.au/eispapers

Part of the Engineering Commons, and the Science and Technology Studies Commons

Research Online is the open access institutional repository for the University of Wollongong. For further information contact the UOW Library: research-pubs@uow.edu.au 


\title{
Reconstructing highly accurate relative stopping powers in proton computed tomography
}

\author{
Abstract \\ Proton computed tomography $(\mathrm{pCT})$ is an evolving tomographic imaging modality with applications in \\ proton and ion therapy. The method allows direct reconstruction of relative stopping power of patient \\ tissues in a 3D-fashion. The PCT collaboration has built first experimental prototypes of PCT scanning \\ systems [1] and has developed approaches to reconstruct proton CT images based on registering the \\ coordinates and water equivalent path length (WEPL) of individual protons traversing the scanned \\ volume. From these data one reconstructs the object boundary (hull) and initial image based on filtered \\ back projection (FBP), calculates a most likely path (MLP) for each proton, and improves the initial image \\ iteratively by solving a large linear system of equations of the form $A x=b$ using an iterative projection \\ algorithm [2].

\section{Disciplines} \\ Engineering | Science and Technology Studies

\section{Publication Details} \\ Schultze, B., Karbasi, P., Giacometti, V., Plautz, T., Schubert, K. E. \& Schulte, R. W. (2015). Reconstructing \\ highly accurate relative stopping powers in proton computed tomography. 2015 IEEE Nuclear Science \\ Symposium and Medical Imaging Conference (NSS/MIC) (pp. 1-3). United States: IEEE.

\section{Authors} \\ Blake Schultze, Paniz Karbasi, Valentina Giacometti, Tia E. Plautz, Keith Schubert, and Reinhard W. Schulte
}




\title{
Reconstructing Highly Accurate Relative Stopping Powers in Proton Computed Tomography
}

\author{
Blake Schultze $^{[1]}$, Paniz Karbasi ${ }^{[1]}$, Valentina Giacometti ${ }^{[2]}$, Tia Plautz ${ }^{[3]}$ Member, IEEE, \\ Keith E. Schubert ${ }^{[1]}$ Senior Member, IEEE, and Reinhard W. Schulte ${ }^{[4]}$ Member, IEEE
}

\section{INTRODUCTION}

$\mathbf{P}$ ROTON computed tomography (pCT) is an evolving tomographic imaging modality with applications in proton and ion therapy. The method allows direct reconstruction of relative stopping power of patient tissues in a 3D-fashion. The pCT collaboration has built first experimental prototypes of pCT scanning systems [1] and has developed approaches to reconstruct proton $\mathrm{CT}$ images based on registering the coordinates and water equivalent path length (WEPL) of individual protons traversing the scanned volume. From these data one reconstructs the object boundary (hull) and initial image based on filtered back projection (FBP), calculates a most likely path (MLP) for each proton, and improves the initial image iteratively by solving a large linear system of equations of the form $\mathbf{A x}=\mathbf{b}$ using an iterative projection algorithm [2].

In this system of equations, the system matrix $\mathbf{A}$ describes the mean effective path length of the $i$ th proton through the $j$ th object voxel [3], $\mathbf{x}$ is the object RSP vector, and $\mathbf{b}$ is the vector of measured WEPL values. In previous work, the initially reconstructed FBP image was thresholded to define the object hull, while more recently we have employed hull detection techniques based on individual WEPL thresholding and space carving for better hull definition [4]. The goal of the current work is to further improve the quality of reconstructed proton CT images. Here, we demonstrate with Geant4 Monte Carlo simulated proton CT data of the Catphan CTP 404 module, that by combining advanced hull detection techniques with the FBP image as the initial iterate, very accurate RSP reconstruction to better than $1 \%$ of the reference value of different phantom insert materials can be achieved.

\section{Methods}

The steps taken in the image reconstruction process are outlined in the diagram shown in Figure 1 and explained in greater detail in the following sections.

[1] Blake Schultze, Paniz Karbasi, and Keith Schubert are with the Department of Electrical and Computer Engineering, Baylor University, Waco, TX 76798, USA, email: Blake_Schultze@ baylor.edu, Paniz_Karbasi@baylor.edu, Keith_Schubert@baylor.edu

[2] Valentina Giacometti is with the Center for Medical Radiation Physics, University of Wollongong, Wollongong, NSW, Australia, email: valentina8giacometti@gmail.com

[3] Tia Plautz is with the Santa Cruz Institute for Particle Physics, University of California, Santa Cruz, Santa Cruz, CA 95064, USA, email: tiaplautz@gmail.com

[4] Reinhard Schulte (rschulte@1lu.edu) is with the Department of Basic Sciences, Loma Linda University Medical Center, Loma Linda, CA 92354 USA

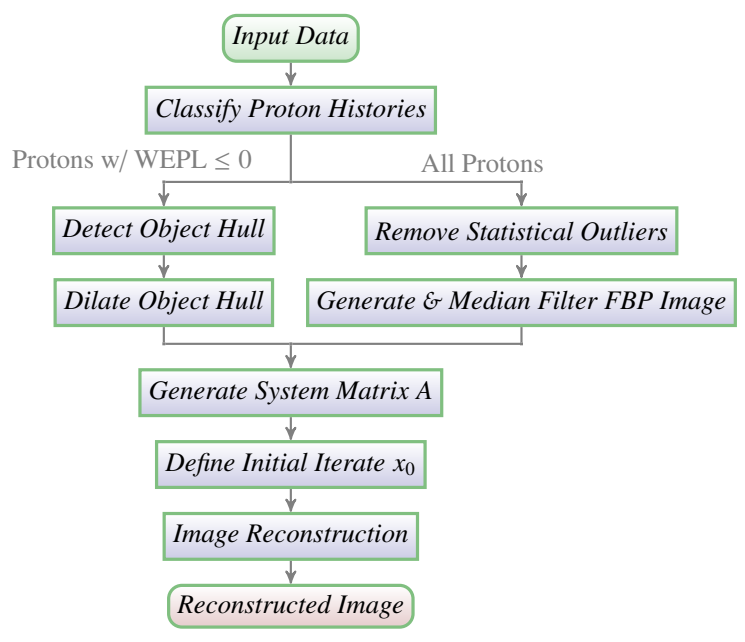

Fig. 1: Reconstruction steps

\section{A. Classify Proton Histories}

To relate the proton energy deposited in the energy detector of the pCT scanner to the water-equivalent path length (WEPL) of the proton in the object, a calibration is performed prior to scanning the object [5]. Since air has a very low RSP $(\approx 0.0013)$, protons passing exclusively through air will generate small WEPL measurements. Hence, the calibrated WEPL measurements can then be used to distinguish between protons that do and do not pass through the object. For this investigation, any proton with WEPL measurement at or below 0.0 was assumed to have missed the object and was then used to generate a convex hull of the object via hull detection [6], [4].

1) Detect and Dilate Object Hull: Hull-detection uses the protons identified as having missed the object to identify regions of space which do not contain any portion of the object being scanned. Beginning with the assumption that the object fills the entirety of the image reconstruction volume, In this work, hull detection was performed using Modified Space/Silhouette Carving (MSC), as this has been shown to generate an accurate convex hull, even if some of the protons used were misidentified as having missed the object without requiring anomalous data to first be removedeven if some of the protons used were misidentified as having missed the object and anomalous data has not yet been removed [6], [4]. It is important to ensure the hull includes all voxels belonging to the object since reconstruction does not update voxels outside the hull and cannot recover any voxels that are missing from 
the hull. Since the most likely voxels to be missing from the hull are from the edge of the object, morphological dilation was performed using a square of side length $l=2 r-1$ as the structural element, thereby expanding the boundary of the object by $r$ voxels; in this investigation, a square of side length $l=3$ was used as the structural element to expand the object boundary by $r=1$ voxels in all directions.

2) Removing Statistical Outliers, Generate FBP Image, and Median Filter FBP Image: While hull detection was being performed, a statistical analysis was performed to identify and remove protons with anomalous relative angle or WEPL measurement due to nuclear scattering or multiple proton pile up events. The remaining protons were used to construct the sinogram representation of the data, which was then used as input to filtered back projection (FBP). The statistical analysis and process leading to the generation of the FBP image is the same approach used in previous investigations and is explained in detail in [4].

The FBP image is typically used to define the initial iterate of iterative image reconstruction, but these often contain streaks and other artifacts that ultimately reduce the quality of the reconstructed image. Although an average filter will reduce or eliminate these artifacts and smooth the image as desired, it will also blur the edges separating regions of differing RSP, an effect that will persist after reconstruction. Thus, a median filter with radius 3 was applied to the FBP image as this provided the desired artifact reduction without degrading edge sharpness.

\section{B. Generate System Matrix A}

A proton passing through the object was used for image reconstruction only if both its entry and exit paths intersected the object hull, defined using the tracker coordinates [7]. The coordinates where these intersections occurred were recorded and then used to calculate the proton's most-likely path (MLP) through the object [8]. The MLP of protons passing through the object were then used to construct the $\mathbf{A}$ matrix as shown in previous studies [3], [4]. Since each proton passes through only a small portion of the reconstruction volume, the resulting A matrix is sparse and to reduce memory usage, only its nonzero elements were stored.

\section{Define Initial Iterate $\mathbf{x}_{\mathbf{0}}$}

The number of iterations of image reconstruction required for convergence of RSP values and their accuracy is heavily dependent on how accurately the initial iterate $\mathbf{x}_{\mathbf{0}}$ can be approximated, so the method used to define it is a vital component of image reconstruction. The median filtered FBP image represents a good approximation of RSP values, but it contains nonzero values for voxels identified as lying outside the object hull. Thus, $\mathbf{x}_{\mathbf{0}}$ is initially defined from the FBP image but the voxels lying outside the hull are then set to an RSP of 0 , preventing these from contributing to image reconstruction calculations and improving the accuracy of reconstructed RSP values.

\section{Image Reconstruction}

The diagonally-relaxed orthogonal projections (DROP) method, with 3200 proton histories per block and relaxation parameter $\lambda=0.0001$, was used to reconstruct a $200 \times 200 \times 32$ image. DROP was configured with relaxation parameter $\lambda=$ 0.0001 and each block contained 3200 proton histories. To capitalize on the parallelism present in the preprocessing and image reconstruction processes, particularly in the MLP and DROP calculations, reconstruction was executed on a compute system with a CUDA enabled graphics processing unit (GPU). Significant computational savings were therefore achieved by performing all parallelizable calculations on the GPU. In cases where the volume of data required for computations exceeded the memory capacity of the GPU, the data was partitioned and computations were then performed on each partition separately.

Given the sparsity of the system matrix $\mathbf{A}$ and initial iterate $\mathbf{x}_{\mathbf{0}}$, calculations involving the elements of these two matrices often result in terms generated by addition of or multiplication by zero. The result of such calculations need not be evaluated explicitly and doing so not only unnecessarily consumes computation time, they are also prone to numerical error. Hence, calculations are restricted to those involving the nonzero elements of these matrices, further improving computational efficiency and reducing numerical error.

\section{INPUT DATA}

The data set used for image reconstruction contained just over 180 million proton events generated by a Geant 4 simulated scan of the CTP404 phantom (Phantom Laboratory, New York NY, USA) with $4^{\circ}$ increments in projection angle. This phantom is a $15 \mathrm{~cm}$ diameter acrylic cylinder and contains 5 small acrylic spheres of radius $2,4,6,8$, and $10 \mathrm{~mm}$ and 8 cylindrical inserts of radius $12.2 \mathrm{~mm}$ composed of different materials (PMP, LDPE, Polystyrene, Acrylic, Delrin, Teflon, and 2-air filled). The acrylic sphere are arranged in a circle around near the center of the phantom and the 8 cylindrical inserts are arranged in a circle near the edge of the phantom.

\section{RESUlTS AND Discussion}

A total of 39,750,201 (out of the initial 180 million) protons entered the object hull and were used for image reconstruction. A total of 12 iterations of DROP were performed and the average total computation time over 5 executions, which includes the time spent reading the data from disk and all preprocessing and reconstruction steps, was 6 minutes and 28 seconds. ImageJ was used to analyze the accuracy of the reconstructed RSP values inside each insert and the results of this analysis are summarized in Table I. The slice used for analysis, shown in Figure 2, was from the middle of the phantom since the small acrylic spherical inserts are most prominent in this slice, resulting in a wider range of WEPL measurements and providing a more challenging test of image reconstruction accuracy. Notice that the \% discrepancy in each of the inserts was well under $1 \%$, a level of accuracy which is important to attain for treatment planning purposes. 
Attaining these highly accurate RSP values by improving the detection of small variations in RSP resulted in higher sensitivity to noise and increased standard deviations. It is a well known fact that the noise increases with each iteration of image reconstruction and total variation superiorization (TVS) schemes were developed to prevent its propagation in successive iterations [9]. Convergence slows considerably as the number of iterations increases and if TVS schemes can be used to prevent noise accumulation, it may be possible to reduce standard deviations and achieve the same level of accuracy with fewer iterations.

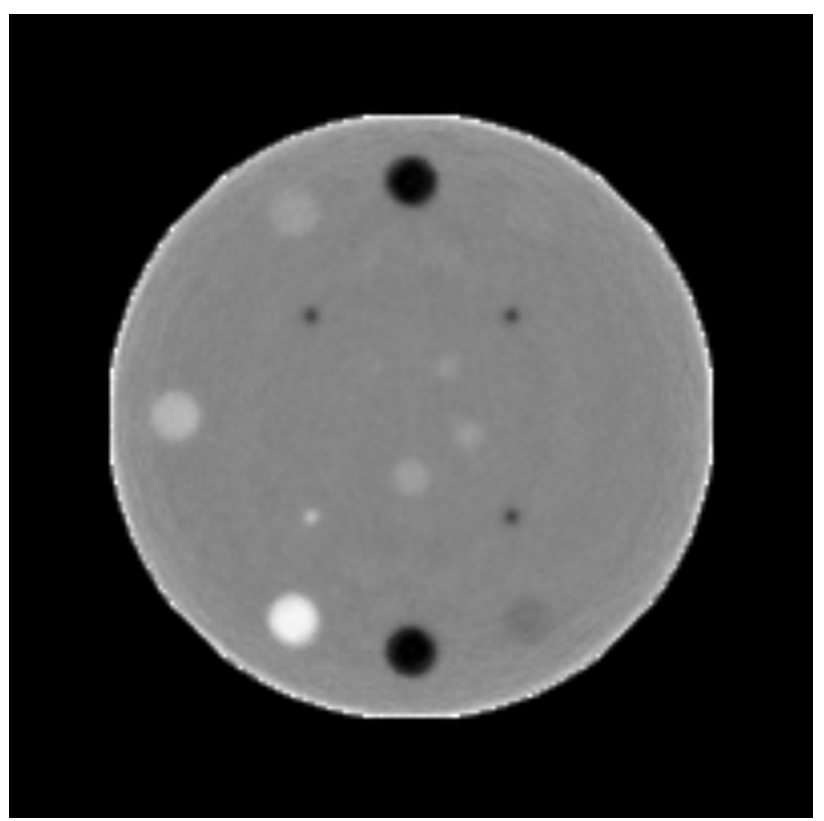

Fig. 2: Reconstructed image after 12 iterations.

TABLE I: Analysis of reconstructed RSP values in each insert

\begin{tabular}{rcccc} 
Insert & Predicted RSP & Mean RSP & \% Discrepancy & Std. Dev. \\
\hline \hline PMP & 0.8770 & 0.8788 & 0.1993 & 0.0201 \\
LDPE & 0.9973 & 0.9990 & 0.1721 & 0.0182 \\
Polystyrene & 1.0386 & 1.0390 & 0.0414 & 0.0178 \\
Acrylic & 1.1550 & 1.1635 & 0.7285 & 0.0199 \\
Delrin & 1.3560 & 1.3532 & -0.2059 & 0.0193 \\
Teflon & 1.8280 & 1.8150 & -0.7161 & 0.0212 \\
\hline \hline
\end{tabular}

\section{SUMmary}

The methods investigated in this work generated promising results given the level of accuracy in reconstructed RSP values, but the number of iterations performed to achieve this accuracy resulted in larger standard deviations than desired. Since TVS schemes were developed to address the issue of noise propagation in successive iterations of iterative image reconstruction, incorporating TVS into the framework presented here should improve the standard deviations obtained in the reconstructed image. Reducing error propagation should also make it possible to achieve convergence in fewer iterations, thereby improving computation time as well. Hence, future studies will investigate the usage of these TVS schemes to determine if standard deviations can be reduced without diminishing the overall accuracy obtained in this work.

\section{ACKOWLEDGEMENTS}

We acknowledge significant contributions to the development of iterative proton CT image reconstruction from Y. Censor (University of Haifa, Israel), and S. Penfold (University of Wollongong, Australia). The research in proton CT is supported by the National Institute of Biomedical Imaging and Bioengineering (NIBIB), and the National Science Foundation (NSF), award Number R01EB013118, and the United States - Israel Binational Science Foundation (BSF) grant nos. 2009012 and 2013003. The content of this paper is solely the responsibility of the authors and does not necessarily represent the official views of the National Institute of Biomedical Imaging and Bioengineering or the National Institutes of Health.

\section{REFERENCES}

[1] H. F.-W. Sadrozinski, R. P. Johnson, S. Macafee, A. Plumb, D. Steinberg, A. Zatserklyaniy, V. Bashkirov, F. Hurley, and R. Schulte, "Development of a head scanner for proton ct," Nucl.Instrum.Meth. A, vol. 699, pp. 205-210, 2013.

[2] S. Penfold, "Image Reconstruction and Monte Carlo Simulations in the Development of Proton Computed Tomography for Applications in Proton Radiation Therapy," Ph.D. dissertation, University of Wollongong, Australia, 2010.

[3] S. Penfold, A. Rosenfeld, R. Schulte, and K. Schubert, "A more accurate reconstruction system matrix for quantitative proton computed tomography," Med. Phys., vol. 36, no. 10, pp. 4511-4518, October 2009

[4] B. Schultze, M. Witt, Y. Censor, K. Schubert, and R. Schulte, "Performance of hull-detection algorithms for proton computed tomography reconstruction," in Infinite Products of Operators and Their Applications, ser. Contemporary Mathematics, S. Reich and A. Zaslavski, Eds., vol. 636. American Mathematical Society, 2015, pp. 211-224.

[5] R. Hurley, R. Schulte, V. Bashkirov, A. Wroe, A. Ghebremedhin, H.-W. Sadrozinski, V. Rykalin, G. Coutrakon, P. Koss, and B. Patyal, "Waterequivalent path length calibration of a prototype proton ct scanner," Med. Phys., vol. 39, pp. 2438-2446, 2012.

[6] B. Schultze, M. Witt, K. Schubert, and R. Schulte, "Space carving and filtered back projection as preconditioners for proton computed tomography reconstruction," in Proceedings of the IEEE Nuclear Science Symposium Medical Imaging Conference, 2012, pp. 4335-4340.

[7] V. Bashkirov, R. Schulte, G. Coutrakon, B. Erdelyi, K. Wong, H. Sadrozinski, S. Penfold, A. Rosenfeld, S. McAllister, and K. Schubert, "Development of Proton Computed Tomography for Applications in Proton Therapy," in APPLICATION OF ACCELERATORS IN RESEARCH AND INDUSTRY: Twentieth International Conference, F. D. McDaniel and B. L. Doyle, Eds., vol. AIP Conference Proceedings Volume 1099. Fort Worth (Texas): American Institute of Physics, August 10-15 2008, pp. 460-463, iSBN: 978-0-7354-0633-9.

[8] R. Schulte, S. Penfold, J. Tafas, and K. Schubert, "A maximum likelihood proton path formalism for application in proton computed tomography," Med. Phys., vol. 35, pp. 4849-4856, November 2008.

[9] S. Penfold, R. Schulte, Y. Censor, and A. Rosenfeld, "Total variation superiorization schemes in proton computed tomography image reconstruction," Med. Phys., vol. 37, pp. 5887-5895, 2010. 